\title{
Response: Does Obesity Affect the Severity of Exercise-Induced Muscle Injury? (J Obes Metab Syndr 2021;30:132-40)
}

\author{
Jooyoung Kim', Jin Hwan Yoon ${ }^{2, *}$ \\ 'Office of Academic Affairs, Konkuk University, Chungju; ${ }^{2}$ Department of Sport Science, College of Life \\ Science and Nano Technology, Hannam University, Daejeon, Korea
}

Received August 21, 2021

Reviewed September 15, 2021

Accepted September 16, 2021

*Corresponding author

Jin Hwan Yoon

https://orcid.org/0000-0001-9026-6416

Department of Sport Science, College of Life Science and Nano Technology, Hannam University, 70 Daedeok-gu, Daejeon 34430, Korea

Tel: +82-42-629-7990

Fax: +82-42-629-8402

E-mail: yoonjh@hnu.kr
In this study, we reviewed the effects of obesity on exercise-induced muscle injuries and the mechanisms involved. As previously described in this study, obesity could be a potential risk factor, increasing the severity of exercise-induced muscle injury. Coronavirus disease 2019 (COVID-19) as a keyword has received the most attention lately. Owing to the government's recommendations and in response to the COVID-19 pandemic, measures such as social distancing, isolation, and home confinement have been implemented; moreover, many restrictions have been placed on daily living activities. Although these restrictions are important to reduce disease transmission, they have also contributed to an increase in obesity by significantly reducing the level of physical activity. ${ }^{1}$ As a result, people have changed their approach to health by focusing their interests and various home fitness exercise options. ${ }^{2}$ Home fitness exercise is a helpful method for maintaining good health during the current situation, and in fact, has been shown to have positive effects physically and psychologically while overcoming dependence on gyms and fitness equipment. ${ }^{3}$

However, the problem with home fitness exercise is that in the absence of an expert, such as an exercise specialist, people often overlook their physical condition or fitness level and perform highintensity exercises. This can result in muscle injury leading to re- duced muscle strength and range of motion, muscle soreness, increase in muscle proteins and enzymes in the bloodstream, and inflammation with reactive oxygen species. ${ }^{4}$ These symptoms are likely to be exacerbated, especially in obese populations. Therefore, when an obese person (whether male or female) starts home fitness exercise, it is necessary to consider ways to reduce muscle injury.

First, it is recommended that preconditioning intervention, such as yoga or foam rolling, be performed before exercise. According to several studies, yoga or foam rolling before exercise can reduce muscle injury. ${ }^{5-7}$ These preconditioning interventions are suitable for home fitness sessions because they can be easily performed anytime and anywhere without expensive equipment or large spaces. Next, after home fitness exercise, it is recommended to consume antioxidant-rich foods, such as tart cherry juice, pomegranate juice, beetroot juice, pineapple juice, and watermelon juice. ${ }^{8}$ These foods contain vitamins, phytochemicals, and other bioactive compounds with anti-inflammatory and antioxidant functions, and are effective at reducing muscle injuries and promoting recovery after exercise. ${ }^{8}$ However, caution should be taken when consuming vitamin $\mathrm{C}$ and E supplements. ${ }^{9}$ Excess vitamins $\mathrm{C}$ and $\mathrm{E}$ can block signaling pathways that should occur in cells after exercise, thus interfering with 
physiological adaptations. ${ }^{10}$

Current studies investigating methods to help reduce muscle injury after exercise have the limitation that they have not been conducted in obese populations. Therefore, their effect on obese people is questionable. These issues need to be considered via well-designed studies in the future.

\section{CONFLICTS OF INTEREST}

Jin Hwan Yoon is an editorial board member of the journal, but he was not involved in the peer reviewer selection, evaluation, or decision process of this article. Otherwise, no other potential conflicts of interest relevant to this article were reported.

\section{AUTHOR CONTRIBUTIONS}

Study concept and design: all authors; drafting of the manuscript: all authors; critical revision of the manuscript: JHY.

\section{REFERENCES}

1. Lim S, Shin SM, Nam GE, Jung CH, Koo BK. Proper management of people with obesity during the COVID-19 Pandemic. J Obes Metab Syndr 2020;29:84-98.

2. Nyenhuis SM, Greiwe J, Zeiger JS, Nanda A, Cooke A. Exercise and fitness in the age of social distancing during the COVID-19 pandemic. J Allergy Clin Immunol Pract 2020;8: 2152-5.
3. Kaur H, Singh T, Arya YK, Mittal S. Physical fitness and exercise during the COVID-19 pandemic: a qualitative enquiry. Front Psychol 2020;11:590172.

4. Peake J, Nosaka K, Suzuki K. Characterization of inflammatory responses to eccentric exercise in humans. Exerc Immunol Rev 2005;11:64-85.

5. Boyle CA, Sayers SP, Jensen BE, Headley SA, Manos TM. The effects of yoga training and a single bout of yoga on delayed onset muscle soreness in the lower extremity. J Strength Cond Res 2004;18:723-9.

6. Sadacharan CM. Pre-exercise strategies: effect of yoga poses on various intensities of eccentric exercise-induced pain and functional limitations. Muscles Ligaments Tendons J 2021;11: 92-100.

7. West JT, Miller WM, Jeon S, Ye X. The Effects of a preconditioning rolling session on subsequent eccentric exercise-induced muscle damage. J Strength Cond Res 2020;34:2112-9.

8. Harty PS, Cottet ML, Malloy JK, Kerksick CM. Nutritional and supplementation strategies to prevent and attenuate exercise-induced muscle damage: a brief review. Sports Med Open 2019;5:1.

9. Higgins MR, Izadi A, Kaviani M. Antioxidants and exercise performance: with a focus on vitamin $\mathrm{E}$ and $\mathrm{C}$ supplementation. Int J Environ Res Public Health 2020;17:8452.

10. Nikolaidis MG, Kerksick CM, Lamprecht M, McAnulty SR. Does vitamin $\mathrm{C}$ and $\mathrm{E}$ supplementation impair the favorable adaptations of regular exercise? Oxid Med Cell Longev 2012; 2012:707941 